\title{
Study on Crack Mechanism of Gas-liquid Inclusions in Quartz Sand under Microwave Heating
}

\author{
Qinglin $\mathrm{Hou}^{1 *}$, Pingfei Chen ${ }^{2}$, Liang Tian ${ }^{3}$, Jiakai Xiao ${ }^{3}$ \\ ${ }^{1}$ Departments of Packing and Materials Engineering, Hunan University of Technology, Zhuzhou, Hunan province,412008, \\ China \\ Corresponding author: Hou Qinglin (1955-), female, chenzhou, Hunan province, China, Hunan University of Technology, \\ $\mathrm{PhD}$.
}

\begin{abstract}
Fourier transform infrared spectrometer (FTIS) and polarizing microscope are utilized to analyze the water molecule content in the gas-liquid inclusions in quartz sand and the micro-morphology change laws of gas-liquid inclusions before and after microwave heating. The experimental results show that when the quartz sand is heated to $600^{\circ} \mathrm{C}$ under microwave, cracks or micro-cracks of gas-liquid inclusions appear at the interface, which causes the $(\mathrm{OH})$-water in the crystal lattice to transform into smaller liquid inclusions. Most gas-liquid inclusions can be removed by continuously increasing the microwave intensity so that the temperature of the quartz particles is as high as $900^{\circ} \mathrm{C}$.Experiments show that the establishment of mathematical model can better reflect the heating behavior of quartz particles in the microwave field, and provide an effective theoretical basis for further study of the influence of these factors on the relationship between internal pressure and tangential tensile stress of inclusions, such as gas-liquid inclusion filling degree, fluid density, homogenization temperature, inclusion radius .
\end{abstract}

\section{Introduction}

With very excellent physical and chemical properties, quartz glass is widely applied to the fields as a nearly irreplaceable top-end material [1], such as optical power, optical communication, semiconductor, $\mathrm{SiO}_{2}$ thin film material, aeronautics and astronautics, military industry, top-end medical appliances.

More than $99 \%$ of superior quartz glass is made from natural crystal In China, natural crystals can reach the requirements through impurity removal only in some regions (such as Lianyungang, Jiangsu, Nanning, Sichuan), unfortunately, this resource has been nearly exhausted in recent 20 years. Thereby, domestic and foreign people have to use natural quartz ores to remove a large number of structurally-composed impurities via an extraordinarily complex process[2-3]. No matter what they do, however, the structural gas-liquid inclusions in the quartz sand fail to be removed thoroughly. Even high-purity quartz sand imported from UNIMIN Company has trace gas-liquid inclusions available so that quartz glass products have the disadvantages of air bubbles and impurities, which have caused the purposes of quartz glass are limited vitally. At present, people first remove trace gas-liquid inclusions from refined so-called high purity quartz sands by hitting lumps, but there are still gas-liquid inclusions which have not been removed after lumping into various quartz glass products. The removal of gas-liquid inclusions is the key to high-end quartz glass raw material and how to thoroughly remove gas-liquid inclusions from refined quartz sand is an outstanding significant theme.

In recent years, the main methods for the removal of gas-liquid inclusions include the thermal crack method, acid-base difference corrosion method and microwave radiation method [4].The first two methods are valid for the removal of gas-liquid inclusions to some extent, but both of them can not completely remove trace gas-liquid inclusions. Microwave radiation method is now the latest process for removing gas-liquid inclusions, but there are few clinging researchers, most of whom only scratch the surface researches. Hou Qinglin et al. [5] made use of the microwave and corrosion effect to remove gas-liquid inclusions in the quartz sand for many years; they found that it was a good effect on the removal of gas-liquid inclusions and thereby discovered the optimal technological condition and relevant control parameters. The result showed that the appropriate microwave radiation could make most gas-liquid inclusions crack or micro-cracks occur on the gas-liquid inclusion interface and then most gas-liquid inclusions could be removed by the subsequent acid corrosion effect. However, they only carried out simple analyses on the crack theory for gas-liquid inclusions under microwave heating, without sufficient knowledge of deep thermal crack mechanism of gas-liquid inclusions under the microwave effect.

$\mathrm{H}_{2} \mathrm{O}$ is, as a strong dipole moment molecule, is the

E-mail: qinglinhou@aliyun.com. Tel: +86 13807331366

Email: qinglinhou@aliyun.com; 274874995@qq.com; 1561435802@qq.com; 874239677@qq.com

TEL:86-18385222157 
most important geological fluid component [6]. Gas-liquid inclusions of unary pure $\mathrm{H} 2 \mathrm{O}$ system are very common in quartz ores and they mainly exist in the form of gas-liquid equilibrium system at normal temperature [7]. According to the selective microwave heating characteristics, $\mathrm{H}_{2} \mathrm{O}$ is a polar substance with a strong ability to absorb microwaves, while the microwave absorption of $\mathrm{SiO}_{2}$ is poorer,so that high temperature inside gas-liquid inclusions occurs momentarily because molecules absorb microwaves to generate friction among vibrating molecules and high pressure appears as well, then the inclusions crack and break through the quartz sand after reaching a certain limit. However, the crack sizes of gas-liquid inclusions are not always the same in quartz sand particles, therefore continuous heating can generate greater internal pressure on the inclusions in different particles, which makes the inclusions completely crack, so as to completely remove the gas-liquid inclusions [8].

A precisely-conditional microwave heating method is adopted in this paper,in order to treat the gas-liquid inclusions in quartz sand and analyze the water molecule

Table 1. ICP-AES Analysis Result (Mass Fraction) of Various Impurities in Quartz Sand Sample

\begin{tabular}{ccccccccccccccc}
\hline Sample & $\mathrm{Al}$ & $\mathrm{B}$ & $\mathrm{Ca}$ & $\mathrm{Cr}$ & $\mathrm{Cu}$ & $\mathrm{Fe}$ & $\mathrm{K}$ & $\mathrm{Li}$ & $\mathrm{Mg}$ & $\mathrm{Mn}$ & $\mathrm{Na}$ & $\mathrm{Ni}$ \\
\hline Raw material & 16.3 & 0.07 & 0.45 & $<0.05$ & $<0.05$ & 0.25 & 0.70 & 0.80 & 0.06 & $<0.05$ & 1.1 & $<0.05$ \\
IOTA & 16.2 & 0.08 & 0.50 & $<0.05$ & $<0.05$ & 0.23 & 0.60 & 0.90 & $<0.05$ & $<0.05$ & 0.9 & $<0.05$ \\
standard & & & & & & & & & & &
\end{tabular}

\subsection{Reagents and Instruments}

(1) Reagents: glycerol, kerosene (reagent level 95\%)

(2) Instruments: polarizing microscope (Leica DM2500P), Fourier transform infrared pectrometer (2000FT•IR SC M ITAR SER ISE), inductively coupled plasma-optical emission spectroscopy (Plasma1000 $\bullet$ ICP - AES), RWS multi-functional microwave experiment furnace, vacuum drying oven, electronic balance and granularity screen $(70 \mathrm{~mm}$ and $140 \mathrm{~mm})$.

\subsection{Experimental Steps}

(1) The quartz particles are screened by using $70 \mathrm{~mm}$ and $140 \mathrm{~mm}$ particle sizes to control the particle size range of the quartz sand particles from 0.1 to $0.2 \mathrm{~mm}$.

(2) Quartz sand sample of $200 \mathrm{~g}$ is weighed and placed in a microwave furnace for heating, the power of sample 1 is set to $1600 \mathrm{w}$ in 50 minutes heated to $600{ }^{\circ} \mathrm{C}$, the power of example 2 is set to $2000 \mathrm{w}$ heated to $900{ }^{\circ} \mathrm{C}$ in 120 minutes.(3) All the samples involved in this paper are placed in the vacuum drying oven under the temperature of $400^{\circ} \mathrm{C}$ for processing for $6 \mathrm{~h}$, so as to remove the water on the surface of quartz sand.

\subsection{Test Method}

The impurity content of the sample was characterized by inductively coupled plasma atomic emission spectrometry. $0.2 \mathrm{~g}$ of the raw material sample and the post-treated sample were respectively placed in a plastic centrifuge tube, and $2 \mathrm{ml}$ of glycerin dispersed sample content change in the gas-liquid inclusions and the micro-morphology change of gas-liquid inclusions in quartz sand .Based on the mathematical calculation model of microwave field model, the formation mechanism of gas-liquid inclusions in quarts sand under microwave radiation is studied, the thermal stress model and internal pressure model of inclusion are discussed, which provide theoretical basis for basic experimental research.

\section{Material and Methods}

\subsection{Raw Material}

Quartz ore in Madagascar region is taken as raw material to prepare quartz sands (purity: about $99.8 \%$, excluding gas-liquid inclusion component), Table 1 indicates ICP-AES analysis result of various impurities in this experimental raw material.

was added dropwise, and the microwave was analyzed by Fourier transform infrared spectrometer. The changes of moisture content in the samples before and after processing were characterized. The shape, size and liquid filling degree of gas-liquid inclusions before and after microwave processing were observed by polarized light microscope. The ore was prepared into a double-sided polished sheet having a thickness of $0.2 \mathrm{~mm}$, and observed under a microscope to confirm the instantaneous temperature at which the inclusions were transferred from two or more phases. The power was set to $2000 \mathrm{~W}$ to record the temperature rise conditions of the quartz sheets for different microwave heating times.

\section{Results}

\subsection{Influences of Microwave Heating on Water Molecule Content in Quartz Sand}

Figure 1 indicates infrared spectroscopy comparison chart of the water content of gas-liquid inclusions in quartz sand before and after microwave processing. Figure 1a indicates the infrared spectrogram of the water content of gas-liquid inclusions in quartz sand in the raw material. Figure $1 \mathrm{~b}$ shows infrared spectrogram of the water content of gas-liquid inclusions in sample 1 after microwave heating with the power of $1600 \mathrm{~W}$ and the time of $50 \mathrm{~min}$ (sample temperature: about $600^{\circ} \mathrm{C}$ ) while figure 1c shows an infrared spectrum of microwave heating with a sample temperature of $900^{\circ} \mathrm{C}$, a power of $2000 \mathrm{~W}$, and a time of $120 \mathrm{~min}$. As shown in Figure 1, after heating up to $600^{\circ} \mathrm{C}$ by microwave as indicated in Figure1(a) and(b), the water molecule infrared absorption 
peak intensity and area of quartz sands greatly increase in the quartz sands not heated by microwave, which specifies that new water molecules are generated available. Literature [9] indicates that new molecules are derived from hydroxyl water $(\mathrm{OH})$ - in quartz lattice. Microwave radiation intensity is strengthened in $\mathrm{b}$ and $\mathrm{c}$ of Figure 1, when the temperature of quartz sand rises from $600^{\circ} \mathrm{C}$ to $900^{\circ} \mathrm{C}$. The infrared absorption peak intensity significantly of quartz sand less than the strength of infrared absorption of raw materials for the water molecules, which suggests that the increase of the intensity of microwave heating to reduce gas liquid inclusions content of quartz sand water molecules).

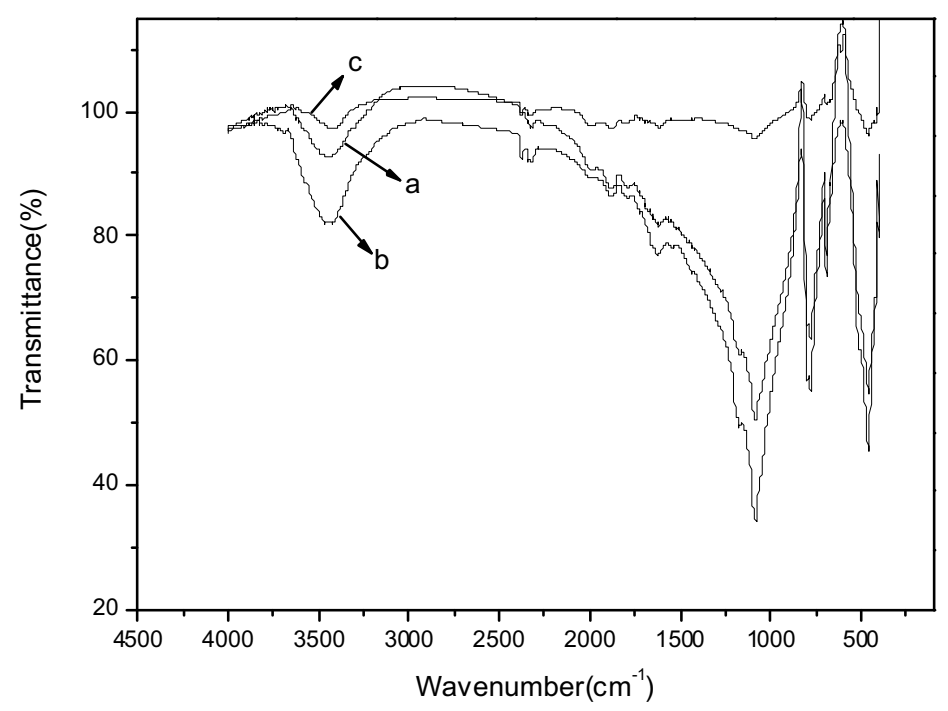

Figure 1. Infrared Spectrogram of Sample before and after Microwave Processing a- No microwave processing; b- Microwave processing $\left(16000 \mathrm{w}-50 \mathrm{~min}-600^{\circ} \mathrm{C}\right)$; $\mathrm{c}-\left(2000 \mathrm{w}-120 \mathrm{~min}-900^{\circ} \mathrm{C}\right)$

\subsection{Influences of Microwave Heating on Microscopic Structures of Gas-liquid inclusions in Quartz Sand}

Gas-liquid inclusions are captured into ore media during formation of ores so as to form some small-volume fluids. Figure 2 indicates microscopic pictures of gas-liquid inclusions in quartz sand particles before and after microwave heating. As shown in Figure 2, there is a certain quantity of larger-volume gas-liquid inclusions in quartz sand raw material in Figure $2 \mathrm{a}$, these inclusions are gas-liquid two-phase inclusions, and the gas-phase part is black while the liquid phase part is light grey. As shown in Figure $2 \mathrm{~b}$ quartz sand heated to $600{ }^{\circ} \mathrm{C}$, some of the volume of a gas liquid inclusions become smaller, especially the volume of a gas phase part greatly reduced.
This shows that cracks occur at gas-liquid inclusion interfaces in this intense microwave radiation, which emits a large amount of gas and impurities, while a certain amount of new small liquid containing tissue is generated, which is consistent with the results of ir spectroscopy in figure 1. Because of microwave heating can produce higher strength and temperature, quartz particle lattice channel expansion of gas-liquid inclusions and water molecules generated under high temperature and pressure can form a large number of new small liquid containing groups of lattice channels [10-11]. According to Figure 2c, the content of gas-liquid inclusions in quartz sand reduces greatly, only with a small quantity of smaller gas-liquid inclusions available, which indicates that microwave heating has a good effect on the removal of gas-liquid inclusions and can even remove gas-liquid inclusions to a greater degree under the acid erosion.
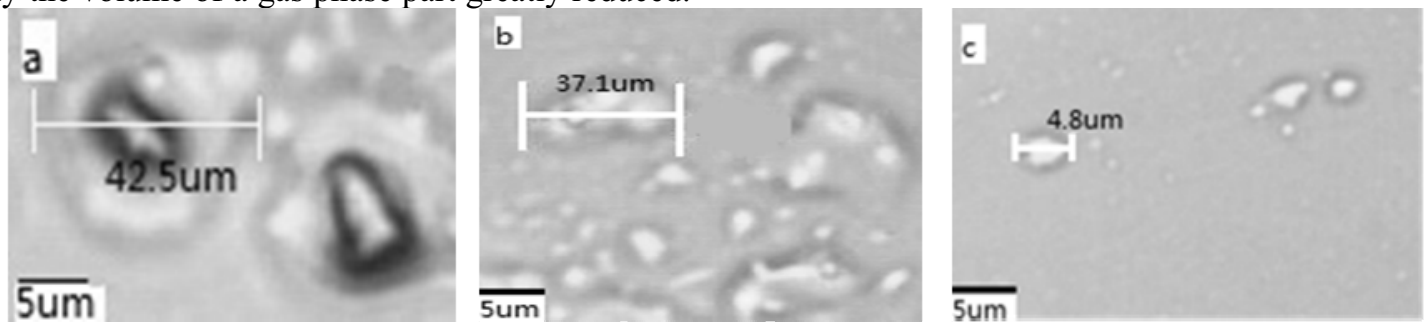

Figure 2. Microscopic Pictures of Samples before and after Microwave Processing a- No microwave processing; b- Microwave processing $\left(1600 \mathrm{w}-50 \mathrm{~min}-600^{\circ} \mathrm{C}\right)$; c- Microwave processing

\section{Discussion}

The experimental above-mentioned researches show that microwave heating can make gas-liquid inclusions in quartz sand crack and generate micro-cracks on the interface.However it is difficult to confirm the effect of factors on the relationship between inclusion crack temperature and crack stress by traditional experiment, 
such as liquid (or gas) filling degree in gas-liquid inclusions, homogenization temperature, inclusion radius, distance from the center of inclusion to the surface of quartz particle, etc. Hence, the crack mechanism of gas-liquid inclusions in quartz sand under the microwave radiation is analyzed theoretically by establishing the mathematical model of gas-liquid inclusions in microwave-cracked quartz sand in this paper, and then a unitary pure water system inclusion is taken as an example to establish the following methods.

\subsection{Establishment of Microwave Field Model}

In the unit time, the energy loss of microwaves in the unit volume of material can be calculated according to formula (1) as follows:

$$
P=2 \pi f \varepsilon_{0} \varepsilon^{\prime \prime} E^{2}
$$

In formula (1), $p$ indicates power density of substance absorbing microwaves (W/ $\mathrm{m} 3) ; f$ indicates microwave frequency $(2450 \mathrm{~Hz}) ; \varepsilon_{0}$ indicates vacuum medium constant $(8.854 \times 10-12 \mathrm{~F} / \mathrm{m}) ; \quad \varepsilon^{\prime \prime}$ indicates dielectric loss factor in complex dielectric constant; $E$ indicates electric field intensity $(\mathrm{V} / \mathrm{m})$.

The waveguide equivalent impedance is calculated by formula (2):

$$
Z_{e}=\frac{b}{a} \sqrt{\frac{u}{\varepsilon}} \frac{1}{\sqrt{1-(\lambda / 2 a)^{2}}}
$$

In formula (2), $a$ indicates long waveguide edge (m); $b$ indicates short waveguide edge $(\mathrm{m}) ; \lambda$ indicates

$$
T_{r}^{k+1}-T_{r}^{k}=\left[\lambda\left(\frac{T_{r+\Delta r}^{k+1}+T_{r-\Delta r}^{k+1}-2 T_{r}^{k+1}}{(\Delta r)^{2}}+\frac{2\left(T_{r+\Delta r}^{k+1}-T_{r}{ }^{k+1}\right)}{r \Delta r}\right)+q_{r}\right] \frac{\Delta t}{\rho \overline{C_{p}}}
$$

The boundary conditions are formula (7), (8) and (9):

Note: the second type of boundary condition is the heat flux $q$ on the boundary in any given moment.

On the most inner layer, namely in the centre of sphere $\mathrm{r}=0$ :

$$
-\left.\lambda \frac{\partial T}{\partial r}\right|_{r=0}=0
$$

On the most outer layer, on the spherical surface $\mathrm{r}=\mathrm{R}$ :

$$
-\left.\lambda \frac{\partial T}{\partial r}\right|_{r=R}=q_{n}
$$

Initial condition:

$$
\left.T(t, r)\right|_{t=0}=T_{0}
$$

wavelength $(\mathrm{m}) ; \quad u$ indicates magnetic permeability $(\mathrm{H} / \mathrm{m})$ and $\varepsilon$ indicates vacuum dielectric constant $(8.854 \times 10-12 \mathrm{~F} / \mathrm{m})$.

The microwave electric field intensity is calculated by formula (3):

$$
E=\frac{\sqrt{P R}}{b}
$$

In formula (3), $E$ indicates field intensity $(V / m)$, $P$ indicates microwave power (W), $R$ indicates equivalent resistance $(\Omega)$ and $b$ indicates short waveguide edge $(m)$.

\subsection{Establishment of 1D Thermal Conduction Model}

Quartz sand particle is supposed as the quartz sand particles are spherical with radius $\mathrm{R}$, as which was shown in Figure 3, the temperature disperses radially in the quartz sand particle, and there are formulas (4) and (5) available at the spherical coordinates:

$$
\rho C_{p} \frac{\partial T}{\partial t}=\frac{\lambda}{r^{2}} \frac{\partial\left(r^{2} \frac{\partial T}{\partial r}\right)}{\partial r}+q_{r}
$$

Namely:

$$
\rho \overline{C_{p}} \frac{\partial T}{\partial t}=\lambda\left(\frac{2}{r} \frac{\partial T}{\partial r}+\frac{\partial^{2} T}{\partial r^{2}}\right)+q_{r}
$$

According to the regional finite difference discrete thought, spherical particles are divided into several micro-element shells radially, with discrete formula (6) as follows:

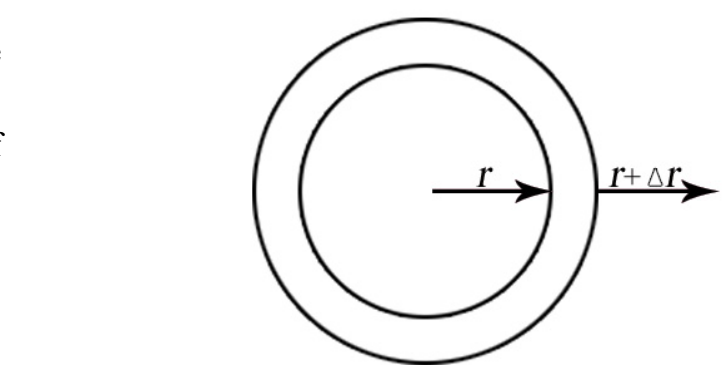

Figure 3. Micro-element Layer of Quartz Particle

In the formula(9), $v_{n}$ indicates volume fraction of surface spherical shell covering the whole sphere, and $\overline{C_{p, n}}$ indicates the average specific heat capacity of the surface spherical shell;

$$
\overline{C_{p, n}}=\frac{H_{T_{n}}-H_{r e f}}{T_{n}-T_{r e f}}=\frac{\int_{T_{r f}}^{T_{n}} C_{p} d T}{T_{n}-T_{r e f}}, T_{r e f} \text { indicates }
$$

the temperature at the reference point, taking $298.15 \mathrm{~K}$, According to Figure 2c, the model of solid specific heat 
capacity $C_{p}$ is referred to physical parameter model in this paper.

\subsection{Thermal Stress Model}

Quartz sand particle is assumed as a sphere to research the radial temperature change conditions, as shown figure.4. The sphere is a hollow one with inside radius of

$$
\left(\sigma_{r}+\frac{d \sigma_{r}}{d r} d r\right)[d \varphi(r+d r)]^{2}-\sigma_{r}(r d \varphi)^{2}-4 \sigma_{\theta}\left[\frac{d \varphi(r+d r+r)}{2}\right] d r \frac{d \varphi}{2}=0
$$

The formula above(10)is expanded and high-order trace quantity is removed to educe the equilibrium equation (11):

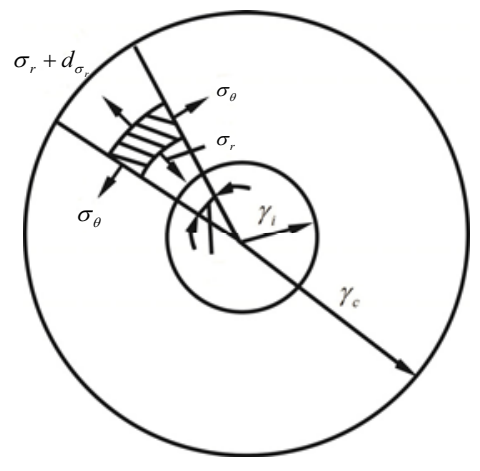

(a) $r_{i}$ and outside radius of $r_{e}$, and the sphere inside is cut into a micro unit formed by planes of inclination of $d_{\varphi}$. In accordance with the force equilibrium of the micro unit, all forces are projected to the connecting line from the center of micro unit to the centre of sphere, to educe formula (10):and the formula (10) is obtained.

$$
\frac{d \sigma_{r}}{d r}+\frac{2\left(\sigma_{r}-\sigma \theta\right)}{r}=0
$$

Figure 4. Micro Units in the Sphere

(a) Hollow Sphere with Inside Diameter of $r_{i}$ and Outside Diameter of $r_{e} \quad$ (b) Micro Units Cut in the Sphere

According to the generalized Hooke's law, here the relationship between stress and strain is shown in formula (12):

$$
\left.\begin{array}{l}
\varepsilon_{r}=\frac{d u}{d r}=\frac{1}{E}\left[\sigma_{r}-2 u \sigma_{\theta}\right]+a t \\
\varepsilon_{\theta}=\frac{u}{r}=\frac{1}{E}\left[\sigma_{\theta}-u\left(\sigma_{r}+\sigma_{\theta}\right)\right]+a t
\end{array}\right\}
$$

The stress express formula (13) can be educed simultaneously from the formula above:

$$
\left.\begin{array}{l}
\sigma_{r}=\frac{E}{(1+u)(1-2 u)}\left[(1-u) \varepsilon_{r}+2 u \varepsilon_{\theta}-(1+u) a t\right] \\
\sigma_{\theta}=\frac{E}{(1+u)(1-2 u)}\left[\varepsilon_{\theta}+u \varepsilon_{r}-(1+u) a t\right]
\end{array}\right\}
$$

$\varepsilon_{r}$ and $\varepsilon_{\theta}$ in formula (13) are replaced by $\frac{d u}{d r}$ and $\frac{u}{r}$ respectively, and the spherical tangential stress formula by deduction and calculation is educed:

$$
\left.\begin{array}{l}
\sigma_{r}=\frac{2 a E}{1-u}\left(\frac{r^{3}-r_{i}^{3}}{\left(r_{e}^{3}-r_{i}^{3}\right) r^{3}} \int \frac{r_{e}}{r_{i}} t r^{2} d r-\frac{1}{r^{3}} \int_{r_{i}}^{r} t r^{2} d r\right) \\
\sigma_{\theta}=\frac{2 a E}{1-u}\left(\frac{2 r^{3}+r_{i}^{3}}{2\left(r_{e}^{3}-r_{i}^{3}\right) r^{3}} \int r_{r_{i}} t r^{2} d r+\frac{1}{2 r^{3}} \int{ }_{r_{i}}^{r} t r^{2} d r-\frac{t}{2}\right)
\end{array}\right\}
$$

\subsection{Internal Pressure Model for Inclusion}

The unitary pure $\mathrm{H} 2 \mathrm{O}$ system uses MRK water state equation (15) corrected by Flowers:

$$
p=\frac{R \cdot T}{V-b}-\frac{a(T)}{T^{0.5} \cdot V \cdot(V+b)}
$$

(1) When the temperature is less than $400^{\circ} \mathrm{C}$,

$$
a(T)=\left(74.174+0.2132 t-4.421 \times 10^{-4} \cdot t^{2}+2.8623 \times 10^{-7} \cdot t^{3}\right) \times 10^{6}
$$

(2) When the temperature is $400 \sim 600^{\circ} \mathrm{C}$, $a(T)=\left(4221-31.227 \cdot t+0.087485 \cdot t^{2}-1.07295 \times 10^{-4} \cdot t^{3}+4.86111 \times 10^{-8} \cdot t^{4}\right) \times 10^{6}$

(3) When the temperature is $600 \sim 1200^{\circ} \mathrm{C}$, 


$$
a(T)=\left(166.8-0.19308 \cdot t+0.1864 \times 10^{-3} \cdot t^{2}-0.71288 \times 10^{-7} \cdot t^{3}\right) \times 10^{6}
$$

(4) When the temperature is higher than $1200^{\circ} \mathrm{C}$,

$$
a(T)=(140-0.05 t) \times 10^{6}
$$

No matter what reason for temperature range above and $\mathrm{b}$ value is changeless; $b=14.6\left(\mathrm{~cm}^{3} / \mathrm{mol}\right)$. In the formula, $P$ indicates pressure $(\times 105 \mathrm{~Pa})$; $\mathrm{t}, \mathrm{T}$ indicates temperature $\left({ }^{\circ} \mathrm{C}, \mathrm{K}\right) ; \mathrm{V}$ indicates molar volume $\left(\mathrm{cm}^{3} / \mathrm{mol}\right) \quad ; \quad \mathrm{R}$ indicates $\left(83.143 \times 10^{5} \mathrm{~cm}^{3} \cdot \mathrm{pa} \cdot \mathrm{mol}^{-1} \cdot \mathrm{K}^{-1}\right)$.

Four models above are programmed by $\mathrm{C}++$ to educe a mathematical model, the calculation model of which is referred to Chart 5 as follows.

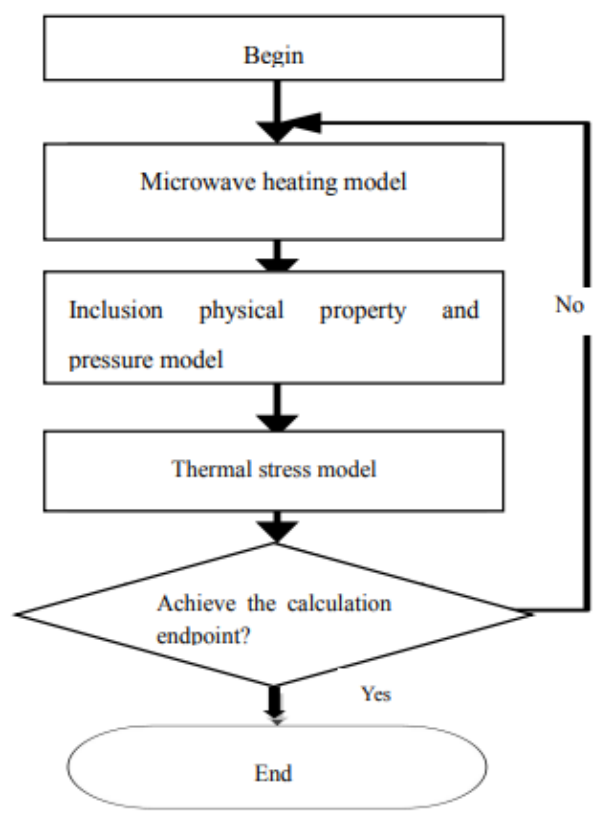

This model can be used to analyze the effect of factors on the relationship between internal pressure and tangential tensile stress of inclusions, such as liquid (or gas) filling degree or fluid density, homogenization temperature, inclusion radius, inclusion size, quartz particle size, etc.

\subsection{Verification of Mathematical Model of Gas-liquid inclusions in Microwave-cracked Quartz Sand}

Because the numerical relationship between factors and inclusion crack fails to be acquired by traditional experiment, such as fluid density, homogenization temperature, inclusion size and so on, the values of microwave time, microwave power and quartz particle temperature are used by experiment to verify the accuracy of the mathematical model presented by this paper.

Figure 6 sets out the modalities of gas-liquid inclusions in quartz lamella sample. As shown in Figure $6 \mathrm{a}$, the shapes of gas-liquid inclusions are approximately oval under microscope, the liquid phase of microscopic hot stage is $F=\frac{V_{L}}{V_{V}+V_{L}}=0.95$, the diameter of gas-liquid inclusion is $37.5 \mathrm{um}$ and that of air bubble is 4.9um. As shown in Figure 6b, after microscopic hot stage heating, this gas-liquid inclusion is evened as liquid phase, with homogeneous temperature of $158^{\circ} \mathrm{C}$.

Figure 5. Model Calculating Flow
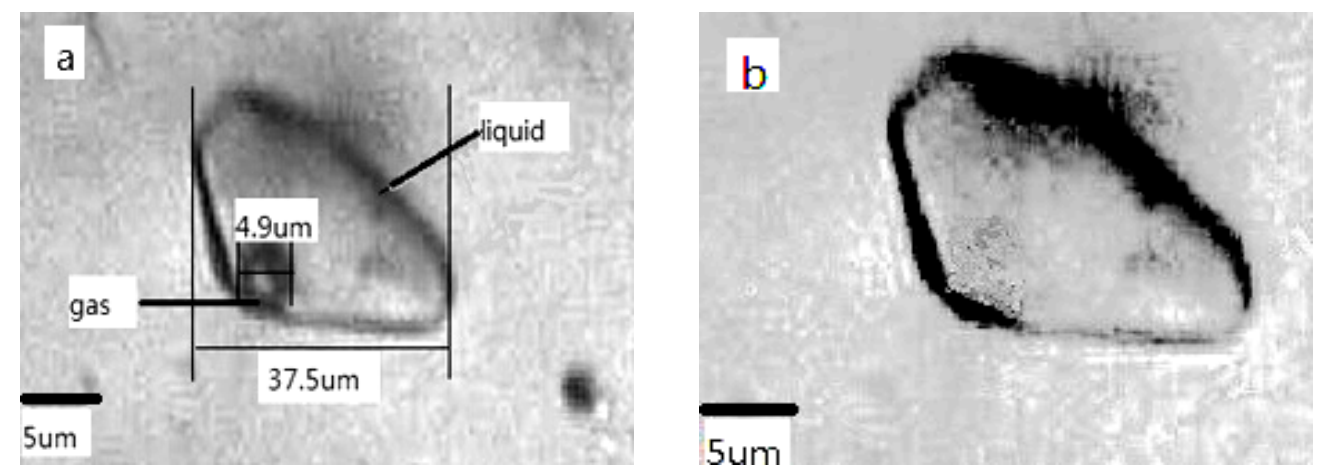

Figure 6. Modalities of Gas-liquid inclusions in the Quartz Lamella a- No heating; b- Microwave hot stage heating

According to Figure 6, gas-liquid inclusion in the quartz lamella sample is irregular. In order for model verification, the gas-liquid inclusion is deeded to be a sphere during the model verification calculation. The radius of the spherical inclusion is $0.0185 \mathrm{~mm}$ and the distance from the center of inclusion to the quartz particle is $0.05 \mathrm{~mm}$. This model is used to calculate the temperature data of quartz particles under the microwave heating, and the setting conditions for the model calculation are referred to Table 2 and the calculated data are compared with the experimental data, the result are shown in Figure 7. 
Table 2. Model Calculating Conditions

\begin{tabular}{cccccc}
\hline Parameter & $\begin{array}{c}\text { Inclusion } \\
\text { radius } \\
(\mathrm{mm})\end{array}$ & $\begin{array}{c}\text { Distance from the } \\
\text { center } \\
\text { of heat source }(\mathrm{mm})\end{array}$ & $\begin{array}{c}\text { Homogenization } \\
\text { temperature }\left({ }^{\circ} \mathrm{C}\right)\end{array}$ & $\begin{array}{c}\text { Liquid } \\
\text { filling } \\
\text { degree }\end{array}$ & $\begin{array}{c}\text { Microwave } \\
\text { power }(\mathrm{w})\end{array}$ \\
\hline $\begin{array}{c}\text { Reference } \\
\text { value }\end{array}$ & 0.0185 & 0.08 & 158 & 0.95 & 2000 \\
\hline
\end{tabular}

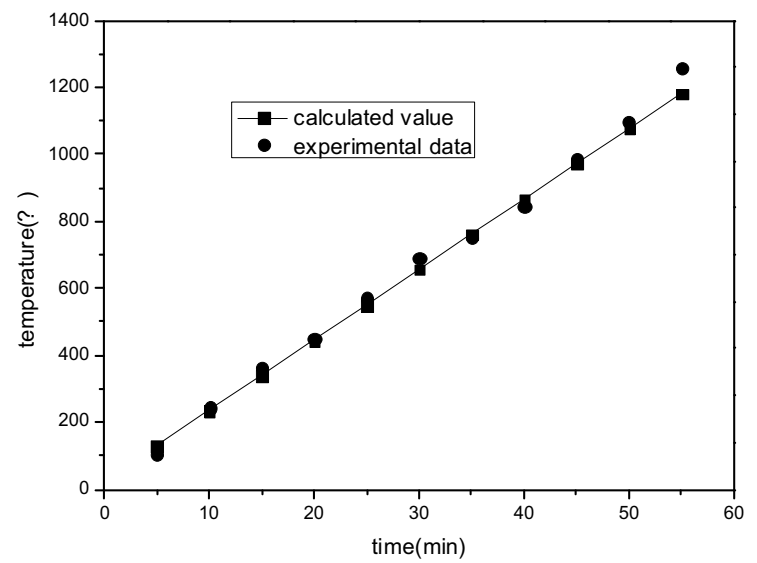

Figure 7 . Calculated Value and Experimental Value

As shown in Figure 7, heating rate of quartz lamellas is constant, which are calculated by crack model for gas-liquid inclusions in quartz sand under microwave heating. Under the condition that physical property parameter and microwave power are known, quartz particle temperature $(\mathrm{T})$ and microwave heating time $(\mathrm{T})$ show a linear relation. According to the experimental result, the temperatures of the quartz lamellas in microwave furnace are distributed on the two sides of this straight line, extremely approaching to the calculated result with smaller deviation. The causes for the deviation are as follows: (1) quartz particles and inclusions are assumed as spheres during the model calculation, and the inclusions are located in the center of quartz particle sphere, which deviates from the actual modalities of actual inclusions. (2) radiuses of quartz particle and inclusion are estimated during the model calculation. (3) the temperature of quartz lamellas measured by microwave infrared thermometer is fluctuant to some extent, so the average value of temperatures measured within $5 \mathrm{~s}$ during the modal calculation is taken as the experimental temperature.

\section{Conclusions}

The calculated result of the crack model of gas-liquid inclusions in microwave heating quartz sand is consistent with the actually-measured value in substance, which indicates that this model is able to better reflect the state change of gas-liquid inclusions heated in the microwave field and it can be used as an effective crack behavior tool when gas-liquid inclusions are heated in the microwave field. In addition, the model can be used to further investigate the effects of factors on the relationship between internal pressure and tangential tensile stress of inclusions, such as fluid (or gas) filling or fluid density in gas-liquid inclusions, homogenization temperature, inclusion radius. The distance from the center of the inclusions to the surface of the quartz particles provides a deep theoretical basis for the study of gas-liquid inclusion cracks in quartz sand under microwave heating.

\section{Acknowledgements}

We would like to acknowledge the National Natural Science Foundation of China (51374103), the National Natural Science Foundation of China (51674114), and the National Natural Science Foundation of China (51174085) for financial support.

\section{References:}

1. León M, et al. Neutron irradiation effects on optical absorption of KU1 and KS-4V quartz glasses and Infrasi 1301[J].Fusion Engineering and Design:2009, 84(7-11):1174-1178.

2. Qinglin Hou, et al.Study on Gas-Liquid Inclusions in Quartz Sand under Microwave Field[J].Advanced Materials Research,2012,(581-582):689-693.

3. ZHANG Shi-xuan. Studis of Eliminating the GasLiquid Wrapped Matter in Qu artz [J].Journal of Jinzhou Normal College (Natural Science Edition):2002, 23(3): 13-14.

4. LI Qing-hai, et al. Impurity Removement from Fine Silica Powder by Thermal Crack Method [J].Nonferrous Metals: 2005, 57 (3): 41-43.

5. YIN Rui-ming, et al. Removal mechanism of 
gas-liquid inclusions in quartz sand under microwave and acid corrosion[J]. Journal of Central South University (Science and Technology), 2014, 45(2): 390 .

6. Frezzotti M L, et al. Raman spectroscopy for fluid inclusion analysis $[\mathrm{J}]$. Journal of Geochemical Exploration, 2012, 112: 1-20.

7. Bodnar R J, et al. Synthetic fluid inclusions in natural quartz. III. Determination of phase equilibrium properties in the system $\mathrm{H}<\mathrm{sub}>2</ \mathrm{sub}>\mathrm{O}-\mathrm{NaCl}$ to $1000^{\circ} \mathrm{C}$ and 1500 bars[J]. Geochimica et Cosmochimica Acta, 1985, 49(9): 1861-1873.

8. HOU Qing-lin, et al. Study on Excellent Mineral Processing for Removal Gas-Liquid Inclusions in Quartz Sand under Microwave and Acid Corrosion[J]. Journal of Hunan University of Technology, 2013 (1): 1-5.

9. ZHOU Yongheng. Studies of quartz glass and hydroxyl groups in raw material[D]. Beijing: China Building Materials Academy, 2002: 173-178.

10. GUAN Shen-jin, et al. An Experimental Study on Synthetic fluid inclusions of $\mathrm{NaCl}-\mathrm{H} 2 \mathrm{O}-\mathrm{CO} 2$ system[J]. Bulletin of Mineralogy, Petrology and Geochemistry, 2012, 31(1): 71-77.

11. LIU Bin. The calculation of the thermodynamic parameters of fluid inclusions softwares and examples[M]. Geological publishing house, Beijing: 2000. 